\title{
Management of laryngeal precancerous lesions
}

\author{
Francesco Dispenza $^{\mathrm{a}, *}$, Alessandro De Stefano ${ }^{\mathrm{b}}$, Donatella Marchese ${ }^{\mathrm{a}}$, Francesco Martines ${ }^{\mathrm{a}}$, \\ Carlo Dispenza ${ }^{a}$ \\ a Dipartimento Biomedicina e Neuroscienze Cliniche - U.O. Otorinolaringoiatria, Università degli Studi di Palermo, Italy \\ ${ }^{\mathrm{b}}$ ENT Institute, Department of Surgical, Clinical and Experimental Sciences, “G.d'Annunzio" University of Chieti and Pescara, Italy
}

\section{A R T I C L E IN F O}

\section{Article history:}

Received 5 April 2011

Accepted 1 August 2011

Available online 20 August 2011

\section{Keywords:}

Larynx

Cancer

Precancerous

Precancerosis

Laryngoscopy

\begin{abstract}
A B S T R A C T
Objective: The identification of precancerous lesions is the basis of an early diagnosis, and of a treatment that allows, in the great part of cases, the preservation of organ functions. The aims of this study were: the evaluation of the less invasive treatment for precancerous lesion of the larynx to minimize the recurrences, the estimation of number of further operation required.

Methods: A prospective study was done on patients with clinical diagnosis of laryngeal precancerosis. The patients were treated by a transoral endoscopic approach with direct microlaryngoscopy (DML) doing an excision-biopsy with cold blade, consisting in excision of the whole visible lesion with vocal ligament preservation.

Results: A recurrence of a clinically evident precancerous lesion was present in $13.2 \%$ of patients that had a laryngeal intraepithelial lesion (LIN) 1 lesion and in 28.95\% of patients that had a LIN 2 lesion. Conclusion: In order to achieve a control of a precancerous lesion, we suggest: excisional biopsy/ subepithelial cordectomy (type 1 cordectomy) for LIN 1 lesions and subligament cordectomy (type 2 cordectomy) in case of LIN 2 cases. In case of recurrences of LIN 1 lesion we suggest directly a type 2 cordectomy.
\end{abstract}

() 2011 Elsevier Ireland Ltd. All rights reserved.

\section{Introduction}

The prevention of neoplastic diseases is realized by identification of mechanism underlying the carcinogenesis and by the knowledge of clinical pictures that indicate a precancerous conditions that may evolve to cancer, although not necessarily.

The WHO classified precancerous lesions as "progressive morphologic alteration of the epithelium because of irritating chronic local factor or referable to a local expression of generalized illness, presenting a higher probability of degeneration into carcinoma with respect to the surrounding mucosa" [1].

The clinical lesions recognized as precancerous in literature are called variously: leukoplakia, erythroplakia, erythrokeratosis, dyskeratosis, white lesion, keratosis, but generally all are determined by a chronic laryngitis. It has now been unanimously recognized that the classification of precancerous lesions must be based on the progression of the specific histopathological characteristics, which in turn considers the grade of epithelial hyperplasia and dysplasia [2].

\footnotetext{
* Corresponding author. Fax: +39 091442084.

E-mail address: francesco.dispenza@gmail.com (F. Dispenza).
}

Kleinsasser described two clinical forms macroscopically detec: the limited epithelial hyperplasia and the hyperplasic chronic laryngitis [3].

In 1974 Bocca et al. identified clinically the precancerous condition in the keratosic epithelial hyperplasia of the vocal cord, distinguished in two macroscopic forms: plane and raised [4].

To standardize the medical terminology on precancerous lesion of the larynx, the terms keratosis or leukoplakia, describing only the clinical condition, should be adopted without histopathological meaning. In fact, several histological features could be found under the same clinical lesion.

The histopathological alterations noticed in the larynx precancerosis are: epithelial hyperplasia, epidermoid metaplasia, acanthosis, keratosis, dyskeratosis, pachydermia, dysplasia.

Hellquist et al. proposed the following classification: (I) hyperplasia with or without low dysplasia; (II) moderate dysplasia; (III) severe dysplasia or in situ carcinoma [5].

The dysplasia is thus considerate an early tumor limited to the epithelial layer, hence Friedmann proposed a classification getting idea from the classification of uterine precancerous lesions [6]. He divided the precancerous lesions into two groups: keratosis without dysplasia and keratosis with dysplasia. The latter was subdivided into three groups according with the grade of dysplasia: LIN 1 (low grade), LIN 2 (moderate grade), LIN 3 (high grade); the term LIN 
indicate "laryngeal intraepithelial lesion". The LIN 3 was identified also with carcinoma in situ (CIS).

The aims of this study were: the evaluation of the less invasive treatment for precancerous lesion of the larynx to minimize the recurrences, the estimation of number of further operation required.

\section{Methods}

A prospective study was done on patients with clinical diagnosis of laryngeal precancerosis in the period 2004-2005. All consecutive patients presenting a vocal cord precancerous lesion were enrolled in the study. The criteria to consider as eligible a patient were: leukoplakic or keratosic lesion of the vocal cord, erytroplakia or erytrokeratosis, normal motility of the vocal cord and the arythenoid, no previous laryngeal surgery, no previous tracheal intubations. The term leukoplakia and keratosis were considered as equal, specifying only the morphological features as plane or raised respect to the normal mucosa. A history was collected with particular attention to risk factor presence (tobacco smoke, works environments, alcohol). All patients enrolled underwent to head and neck examination, fiberoptic laryngoscopy, and vocal performance questionnaire (VPQ: 12 questions with a score of $1-5$ ). The patients were treated by a transoral endoscopic approach with direct microlaryngoscopy (DML) doing an excisionbiopsy with cold blade, consisting in excision of the whole visible lesion with vocal ligament preservation. A histopathological examination was obtained in all cases classifying the results according with Friedmann. The patients were followed-up every 2 months for the first year and every 6 months in subsequent years. The clinical follow-up was done with fiberoptic laryngoscopy.

In case of diagnosis of early glottic cancer after the first operation the patients are treated by a transmuscular cordectomy (type 3) under DML. In case of recurrence of the disease during follow-up we adopt a type 4 cordectomy under DML. All patients exhibiting a cancer recurrence after the first treatment are managed with open surgery as fronto-lateral laryngectomy and supracricoid laryngectomy (Fig. 1).

The preoperative records, the data obtained after surgery and the results after follow-up were analyzed statistically.

\section{Results}

The patients examined and treated included in the study were 232. The mean age was 59 years (range $34-82$ years). The male/ female ratio was $8: 1$. $86 \%$ of patients were smokers. $43 \%$ of patient resulted alcohol consumer of more than $30 \mathrm{ml} /$ day. The patients were firstly classified according to the clinical diagnosis: plane keratosis in 81 cases (Fig. 2), raised keratosis in 80 cases (Fig. 3), and erythrokeratosis in 71 cases. The surgical operation consisted in the excision of the whole visible lesion with preservation of the vocal ligament. The histopathological examination showed a variable grade of dysplasia that was classified in accordance with Friedmann: LIN 1 in 53 cases, LIN 2 in 76 cases and LIN 3/CIS in 46

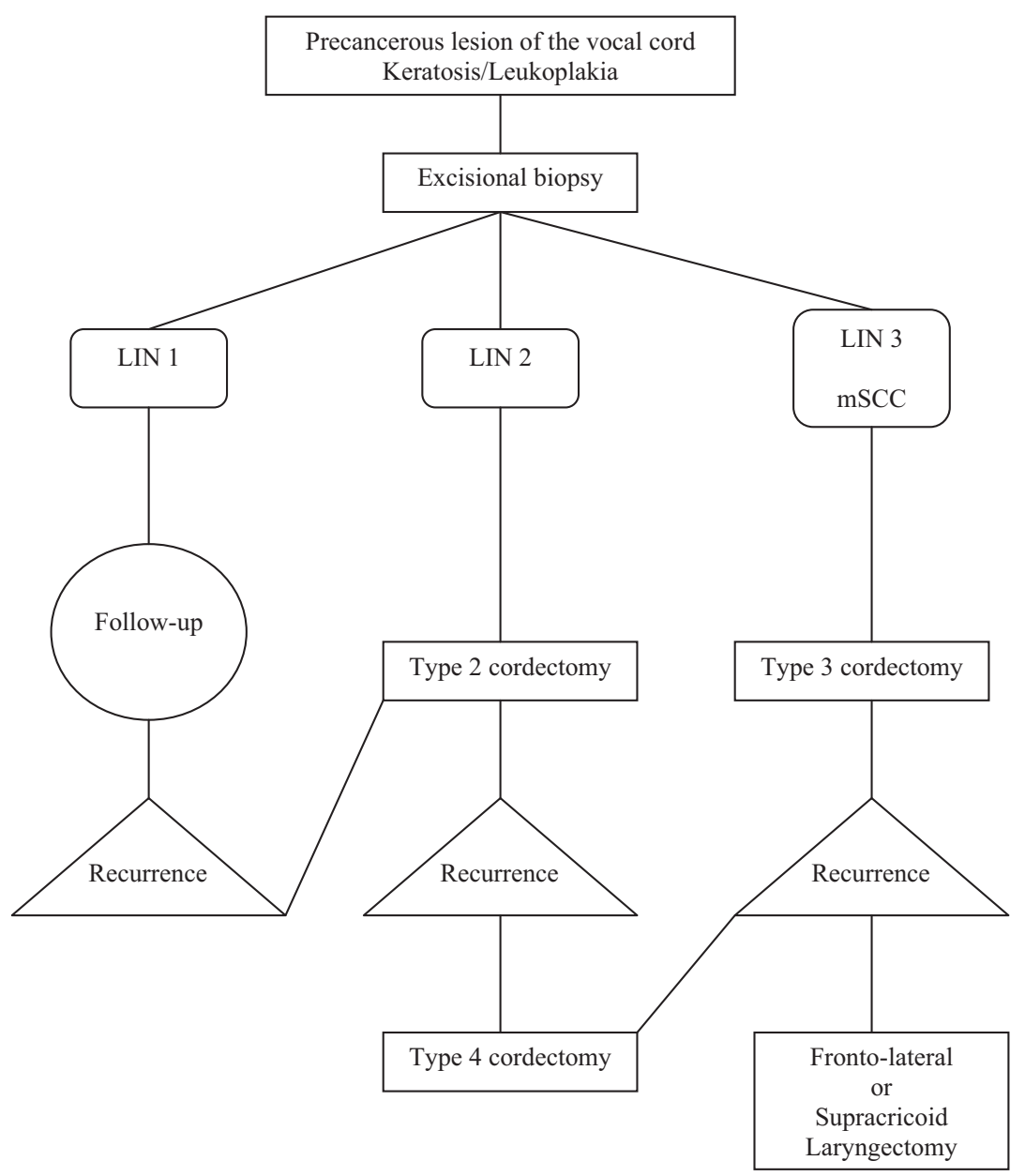

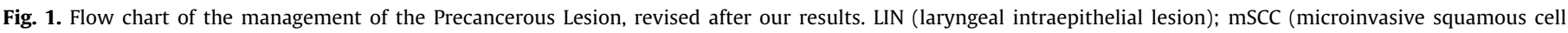
carcinoma). 


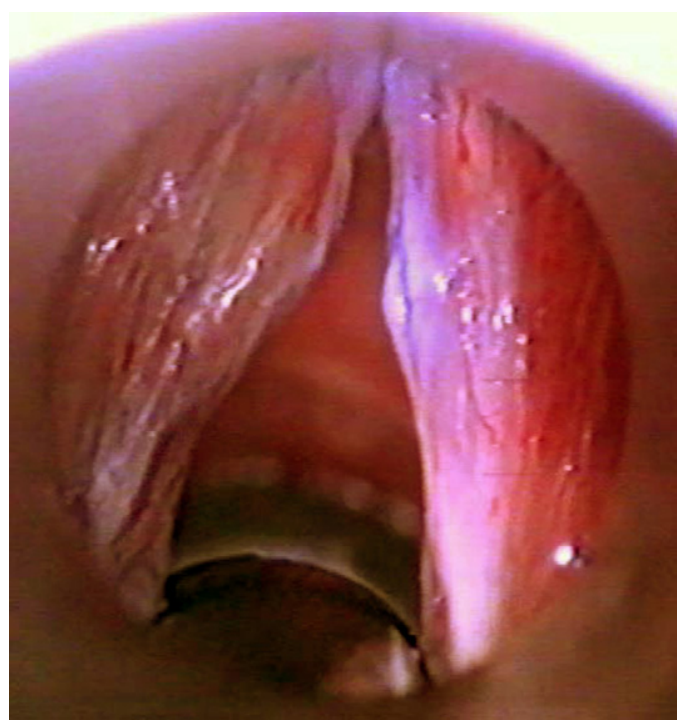

Fig. 2. Direct laryngoscopic views of a plane keratosis of the right vocal cord, the lesion involve the medial aspect of the vocal cord.

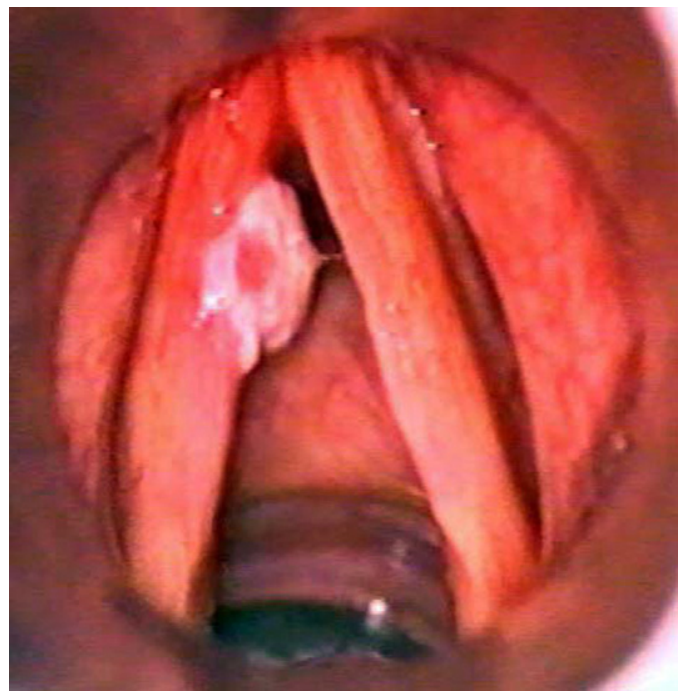

Fig. 3. Direct laryngoscopic view of a raised keratosis of the left vocal cord.

cases, and a microinvasive squamous cell carcinoma (mSCC) was identified in 57 cases (Table 1 ).

A stratification of histopathological findings with the clinical features revealed the incidence of LIN 3/CIS in relation with clinical appearance: 16 cases among plane keratosis, 21 cases among raised keratosis, and 9 cases among erythrokeratosis. The 57 patients with mSCC were stratified as follows: 13 patients with

Table 1

Correlation between clinical features of precancerous lesions and histopathology. The clinical features are often not indicative of the underlying malignancy. LIN (laryngeal intraepithelial lesion); mSCC (microinvasive squamous cell carcinoma).

\begin{tabular}{lllll}
\hline & Plane keratosis & Raised keratosis & Erythrokeratosis & \\
\hline LIN 1 & $23(9.9 \%)$ & $13(5.6 \%)$ & $17(7.3 \%)$ & $53(22.8 \%)$ \\
LIN 2 & $29(12.5 \%)$ & $26(11.2 \%)$ & $21(9.1 \%)$ & $76(32.7 \%)$ \\
LIN 3 & $16(6.8 \%)$ & $21(9.1 \%)$ & $9(3.9 \%)$ & $46(19.8 \%)$ \\
mSCC & $13(5.6 \%)$ & $20(8.6 \%)$ & $24(10.3 \%)$ & $57(24.6 \%)$ \\
& $81(34.9 \%)$ & $80(34.5 \%)$ & $71(30.6 \%)$ & 232 \\
\hline
\end{tabular}

Table 2

Recurrence rates in patients that had low and mild dysplasia.

\begin{tabular}{lll}
\hline & LIN 1 (53) & LIN 2 (76) \\
\hline $\begin{array}{l}\text { Recurrence of precancerous lesion } \\
\begin{array}{l}\text { Cancer development among } \\
\text { LIN1-2 patients }\end{array}\end{array}$ & 7 cases (13.2\%) & 22 cases (29\%) \\
\hline
\end{tabular}

plane keratosis, 20 patients with raised keratosis and 24 patients with erythrokeratosis (Table 1 ).

The analysis of the VPQ scores showed a mean preoperative value of 32.5 points (SD 9.1), while the follow-up test at 3 months showed a mean value of 18.4 (SD 7.1).

The overall incidence of an early glottic carcinoma in case of clinically diagnosed precancerous lesion was of $24.6 \%$ (Table 1 ).

The patients with diagnosis of early glottic cancer after the excision-biopsy underwent to a further operation of total cordectomy type 3 by a transoral endoscopic approach with direct microlaryngoscopy. The patients with diagnosis of LIN 1 and LIN 2 were strictly followed-up.

After 5 years of follow-up we recorded the incidence of the recurrence in all patients. A recurrence of a clinically evident precancerous lesion was present in $13.2 \%$ of patients that had a LIN 1 lesion and in $29.0 \%$ of patients that had a LIN 2 lesion (Table 2). The patients exhibiting recurrence were treated surgically with a type 4 cordectomy. Among recurrences of keratosic lesion, 12 (9.3\%) had a diagnosis of carcinoma after the second treatment; all these patients had a previous LIN 2 lesion. In the group of patients with a previous early glottic cancer, and treated initially with type 3 cordectomy, a recurrence was observed in 5 patients (4.9\%), these patients underwent to Dispenza's modified fronto-lateral laryngectomy [7] or supracricoid laryngectomy.

\section{Discussion}

The laryngeal cancer represents about $1-2 \%$ of all malignant tumors. In $90 \%$ of cases carcinomas develop from precancerous epithelial lesions [8]. Prompt treatment after an early diagnosis is capable to prevent the development of an invasive neoplasm and the consequent recourse to more invasive laryngeal surgery [3,9].

The theory of the transformation of laryngeal keratosis into carcinoma, which occurs through progressive modifications of normal epithelium in keratosis without dysplasia, or the point of degenerating into carcinoma in situ, is confirmed by epidemiological data and the tendency to relapse to a more advanced stage of dysplasia [8]. The concept of pre-invasive lesion has more correctly replaced that of precancerous lesion. This idea came up because carcinoma in situ is classified in the group of severe dysplasia progressing from an intraepithelial neoplasm; consequently a carcinoma in situ should not be considered as a precancerous lesion but as a pre-invasive lesion.

The carcinoma in situ is characterized by a higher incidence of recurrences of both dysplasia and infiltrating carcinoma as showed by some Authors [10-12], and for that reason it should be considered separately from simple dysplasia in term of prognosis and classification [12].

We pointed out elsewhere that most of malignant tumors of the larynx develop from precancerous lesions [13]. As described by Gallo et al. the interval between the first diagnosis of a laryngeal dysplasia and the discovery of the malignant tumor seems to be correlated with the grade of dysplasia [14]. In fact, is a common consideration that the evolution of preinvasive lesion to infiltrating disease could be prevented by an early diagnosis and an accurate treatment.

As reported by several study, the recurrence of a dysplastic lesion ranges between 9.5 and $46.4 \%$, and the type of treatment 
Table 3

Treatment of precancerous lesion and relative recurrence rate of some Authors. The recurrence rate seems to be unlinked to the approach selected.

\begin{tabular}{|c|c|c|c|c|c|c|c|}
\hline Authors & Patients & Surgery & LIN 1 & Recurrence & LIN 2 & Recurrence & SCC \\
\hline Gallo & 84 & Cordectomy Type 1 and 3 & 56 & $14(25 \%)$ & 28 & $13(46 \%)$ & 10 \\
\hline Plch & 88 & Cordectomy Type 1 & 63 & $9(14 \%)$ & 25 & $3(12 \%)$ & 1 \\
\hline Leirens & 32 & Cordectomy Type 1 & 25 & $3(12 \%)$ & 7 & $1(14 \%)$ & 1 \\
\hline Minni & 58 & Cordectomy Type 1 and 2 & 32 & $4(12 \%)$ & 26 & $1(8 \%)$ & \\
\hline Ricci & 78 & Excision Biopsy & 42 & $4(10 \%)$ & 36 & $6(17 \%)$ & 5 \\
\hline
\end{tabular}

was not determinant to the final results $[8,15-18]$ (Table 3$)$. The excision biopsy alone does not seem to present higher recurrence rates compared to simple cord stripping or type 1 cordectomy [16]. For this reason we preferred to adopt a more conservative surgery in case of suspect lesions limited to a little portion of the vocal cord. The excision biopsy allows also performing a single intervention to reach a good result in term of avoiding recurrence.

As easily imaginable, a lower recurrence rate is achieved with a more aggressive treatment and, if similar functional results were obtained after more aggressive treatments (type 2 cordectomy), this would seem to suggest a more radical surgical treatment even for mild forms of dysplasia.

In our opinion this approach may be sometimes questionable, because aggressive treatments without a histopathological confirmation could be overtreatments.

The follow-up is, however, a crucial step after the surgery to prevent invasive disease in case of recurrence, monitoring the patients for the months and years after surgery, especially those with higher grade of dysplasia.

The control of the risk factors covers the same importance of the postoperative follow-up, because $80 \%$ of recurrences occurred in patients who continued alcohol consuming or smoking [8]. The presence of gastroesophageal reflux is now considered also between risk factors of laryngeal dysplasia recurrence [19], and it should evaluated and treated taking into consideration that about $30 \%$ of subjects are asymptomatic.

Among patients with recurrent keratosic laryngeal lesion, we noted a cancer development in 12 (9.3\%) cases, all linked to a LIN 2 lesion. Our recurrence rate (13.2\% for LIN1 and $29.0 \%$ for LIN2) was similar to those reported in literature as Table 3 shows. In 5 patients out from those with early glottic cancer and treated by type 3 cordectomy we recorded a recurrence of cancer.

The intriguing finding that emerges is that in cases of recurrence after cordectomy in moderate dysplasia we have not malignancy development. The low dysplasia (LIN 1) managed with conservative techniques as excision biopsy and type 1 cordectomy, may present some recurrence rate, while surprisingly this does not happen in more severe forms. This is due to a more extensive resection as type 2 cordectomy that is adopted for laryngeal preinvasive lesions that clinically, or histopathologically when available, look as more aggressive.

However the clinical appearance of the preinvasive laryngeal lesion is not related to the final histopathology results as showed by our series. This indication suggests that it is very difficult to predict the recurrence and thus the needs of further treatment only by a clinical feature.

The low recurrence rate observed in case of clinically more aggressive lesion (that histologically resulted as LIN 3, Cis or microinvasive carcinoma) is clearly linked to the more aggressive treatment performed as first approach. The LIN 2 lesion is, thus, the precancerous form that may cause the higher rate of recurrence due to a less aggressive treatment, because is often undistinguishable clinically from a LIN 1 lesion. The lesson learned from this study should be that in case of LIN 2 lesion different treatment strategy could be indicated. In our opinion the way adoptable are two: a first biopsy to ascertain the grade of dysplasia before the excision or a further surgical treatment in case of LIN 2 evidence after first examination.

\section{Conclusions}

In order to achieve a control of a precancerous lesion, minimizing the recurrence rate and preserving a good voice quality, we suggest: excisional biopsy/subepithelial cordectomy (type 1 cordectomy) for LIN 1 lesions and subligament cordectomy (type 2 cordectomy) in case of LIN 2 cases. In case of recurrences of LIN 1 lesion we suggest directly a type 2 cordectomy. The physician should keep in mind that the control of risk factor could reduce the recurrence rate. A prolonged follow-up is absolutely necessary.

\section{References}

[1] WHO. Collaborating center for oral precancerous lesions. Definition of leukoplakia and related lesions: an aid to studies on oral precancer. Oral Surg 1987;46:518-39.

[2] Pallestrini EA, Borasi F, Garaventa G, Parmegiani A. Laryngeal precancerous conditions. Acta Otorhinolaryngol Ital 1991;33:7-17.

[3] Kleinsasser O. Die Klassiffcation und diVerential diagnose der epithelyperplasien der Kehlkopfschleimhaut auf Grund Histomorphologischer Merkmale. Zschr Laryng Rhinol 1963;42:339-62.

[4] Bocca E, Leonardelli GB, Micheu C, Pignataro O. Gli stati precancerosi della laringe. Nuovo Arch Ital Otol 1974;2:287-305.

[5] Hellquist H, Lundgren J, Oloffson J. Hyperplasia keratosis, dyslasia and carcinoma in situ of the vocal cords, a follow-up study. Clin Otolaryngol 1982;7: $11-27$.

[6] Friedmann I. Precancerous lesions of larynx. Can J Otolaryngol 1987;22: 311-52.

[7] Dispenza F, Saraniti C, Mathur N, Dispenza C. Modified fronto-lateral laryngectomy in treatment of glottic T1(a-b) cancer with anterior commissure involvement. Auris Nasus Larynx 2010;37:469-73.

[8] Minni A, Barbaro M, Rispoli G, Diaferia F, Bernardeschi D, Filipo R. Treatment with laser $\mathrm{CO}_{2}$ cordectomy and clinical implications in management of mild and moderate laryngeal precancerosis. Eur Arch Otolaryngol 2008;265: 189-93.

[9] Malzahn K, Dreyer T, Glanz H, Arens C. Autofluorescence endoscopy in the diagnosis of early laryngeal cancer and its precursor lesions. Laryngoscope 2002;112:488-93.

[10] Ngoyen C, Naghibzadeh B, Black MJ, Rochon L, Shenouda G. Carcinoma in situ of the glottic larynx: excision or irradiation? Head Neck 1996;18:225-8.

[11] Stenersen TC, Hoel PS, Boysen M. Carcinoma in situ of the larynx: results with different methods of treatment. Acta Otolaryngol 1988;449:1-133.

[12] Blackwell KE, Calcaterra TC, Fu Y. Laryngeal displasia: epidemiology and treatment outcome. Ann Otol Rhinol Laryngol 1995;104:596-602.

[13] Salzano FA, Dispenza F, Speciale R, Dispenza C. Larynx carcinoma epidemiology. In: Salami A, editor. Identificazione dei marcatori prognostici nel tumore della laringe. Torino: Minerva Medica; 2009. p. 27-31.

[14] Gallo A, de Vincentiis M, Della Rocca C, Moi R, Simonelli M, Minni A, et al. Evolution of precancerous laryngeal lesion: a clinicopathologic study with long-term follow-up on 259 patients. Head Neck 2001;23:40-7.

[15] Sadri M, McMahon J, Parker A. Management of laryngeal dysplasia: a review. Eur Arch Otorhinolaryngol 2006;263:843-52.

[16] Ricci G, Molini E, Faralli M, Simoncelli C. Retrospective study on precancerous laryngeal lesion: long-term follow-up. Acta Otorhinolaryngol Ital 2003;23: 362-7.

[17] Plch J, Par I, Navratilova I, Blahova M, Zavadil M. Long term follow-up of laryngeal precancer. Auris Nasus Larynx 1998;25:407-12.

[18] Leirens J, Vidts G, Schmelzer B, Delle Faille D, Katz S, Van Cauwenberge P. Premalingnant lesion of the vocal fold: a retrospective study of 62 cases. Acta Otolaryngol 1997;117:903-8.

[19] Peretti G, Piazza C, Berlucchi M, Cappiello J, Giudice M, Nicolai P. Pre-and intraoperative assessment of mid-cord erythroleukoplakias. A prospective study on 52 patients. Eur Arch Otorhinolaryngol 2003;260:525-8. 